\title{
GRUPO TERAPÊUTICO EM FONOAUDIOLOGIA: REVISÃO DE LITERATURA
}

\author{
Therapeutic Group in Speech Language and Hearing Sciences: \\ literature review
}

\author{
Vanessa Veis Ribeiro ${ }^{(1)}$, Ivone Panhoca ${ }^{(2)}$, Ana Paula Dassie-Leite ${ }^{(3)}$, Maria Fernanda Bagarollo ${ }^{(4)}$
}

\begin{abstract}
RESUMO
Tema: grupo terapêutico fonoaudiológico. Objetivo: revisar, de maneira sistemática, pesquisas advindas de todas as áreas da Fonoaudiologia que envolveram abordagens grupais, na realidade brasileira. Foi realizada busca nos bancos de dados das bases SciELO e LILACS no período de 2005 a 2010. Foram selecionados os estudos cujos conteúdos dos resumos relacionavam-se com o objetivo da presente pesquisa. Como forma de categorização dos dados, optou-se pela análise dos seguintes aspectos: público-alvo, ano de publicação e área da Fonoaudiologia envolvida. Conclusão: observase um número restrito de publicações referentes ao tema (28 artigos). A maior parte dos trabalhos foi realizada com público adulto, seguido de grupos de crianças, adolescentes e idosos, respectivamente. Foi baixo o índice de artigos envolvendo grupos de familiares. Dentre as áreas da Fonoaudiologia, a de Linguagem tem o maior número de publicações envolvendo grupos, seguida pelas áreas de Voz e Audiologia. Em relação ao ano de publicação, observou-se que, de maneira geral, tem havido decréscimo do número de publicações sobre o assunto desde o ano de 2007. Concluiu-se que é restrito o número de publicações sobre grupo terapêutico na área de Fonoaudiologia. Considera-se que além de novos estudos sobre a prática grupal, outros trabalhos de revisão devam assumir a análise de categorias como aspectos metodológicos, estratégias de atuação e resultados obtidos nos processos terapêuticos grupais.
\end{abstract}

DESCRITORES: Processos Grupais; Fonoaudiologia; Fonoterapia; Prática de Grupo; Terapêutica

\section{INTRODUÇÃO}

Historicamente a terapia fonoaudiológica tem caráter individual, por ser baseada no modelo

(1) Acadêmica do curso de Fonoaudiologia da Universidade Estadual do Centro-Oeste - UNICENTRO, Irati, PR, Brasil.

(2) Fonoaudióloga Clínica; Mestre e Doutora em Lingüística pelo IEL/UNICAMP; Docente do Programa de Pós-Graduação Stricto Senso da Universidade Mogi das Cruzes.

(3) Fonoaudióloga; Professora Assistente do Departamento de Fonoaudiologia da Universidade Estadual do Centro Oeste/UNICENTRO, Irati, PR, Brasil; Mestre em Saúde da Criança e do Adolescente pela Universidade Estadual de Campinas.

(4) Fonoaudióloga; Professora Colaboradora do Departamento de Fonoaudiologia da Universidade Estadual do Centro Oeste - UNICENTRO, Irati, PR, Brasil; Mestre em Educação pela Universidade Metodista de Piracicaba; Doutora em Saúde da Criança e do Adolescente pela Universidade Estadual de Campinas - UNICAMP.

Conflito de interesses: inexistente médico curativo. Porém, nos últimos anos alguns profissionais que atuam com a prática de terapia fonoaudiológica começaram a desenvolver trabaIhos sob a perspectiva do atendimento coletivo. A opção terapêutica individual baseia-se em uma visão advinda da medicina que acredita nas patologias como formas de desvios e entende a clínica como espaço de 'cura'. Desta maneira, muitas vezes deixa de lado a percepção que o paciente tem sobre sua alteração e a interferência dela em seu cotidiano e inserção social. Nesse modo de trabalho são priorizados exercícios e orientações que se chocam com as reais condições de vida dos pacientes ${ }^{1,2}$.

Os avanços nas pesquisas em Fonoaudiologia têm gerado novos questionamentos e demandas, exigindo aprofundamento, resignificação e reorganização das técnicas utilizadas na própria atuação ${ }^{1,3}$. Neste contexto, surge a prática grupal, que vem sendo vista, na Fonoaudiologia, como educativa e 
terapêutica. A terapia fonoaudiológica em grupo é considerada muito valiosa pelos profissionais que a adotam, por proporcionar a construção conjunta de conhecimento entre os sujeitos e as trocas de experiências, modificando a visão dos indivíduos e propiciando as (re) significações dos processos patológicos ${ }^{1,2}$

A terapia em grupo foi proposta primeiramente devido ao aumento da demanda de pacientes ${ }^{3,4}$. Porém, a motivação para esse tipo de prática está ultrapassada e tem sido substituída pela percepção de que a atuação em grupo pode ser uma importante ferramenta de intervenção $0^{1,3}$.

O prazer na convivência em grupos é inerente à condição de homem, podendo esse ser um fato que motive a participação dos sujeitos nas terapias grupais. Os indivíduos podem desenvolver vínculo de amizade para com os demais participantes do grupo, sentindo-se, assim, acolhidos. Com o tempo, por meio do vínculo terapêutico, eles passam a aderir com maior facilidade as atividades terapêuticas propostas. Tal adesão permite e favorece as trocas intersubjetivas, a inclusão, autopercepção, autoconhecimento, e as expressões de afeto, tornando-se um veículo para o processamento de informações ${ }^{3}$.

Os critérios para composição do grupo, normalmente, são feitos sem levar em conta a patologia, mas sim faixa etária, sexo e contexto profissional dos pacientes. Consideradas as especificidades da intervenção grupal, essa forma de atuação demanda uma formação específica e aprofundada por parte do profissional ${ }^{3,4}$.

Uma das funções do grupo terapêutico é administrar a ansiedade comum a todos os membros, que adquire características particulares a cada um dos participantes. Por isso, quando bem administrada, passa a ser um fator propulsor positivo. Essa troca de experiência entre os integrantes, paulatinamente, ameniza a heterogeneidade do grupo, fazendo com que este se torne mais homogêneo ${ }^{1,3}$.

Muitos profissionais não sabem justificar o motivo que os leva a realizar o trabalho fonoaudiológico em grupo, e, ao mesmo tempo, levantam questionamentos a respeito da efetividade deste tipo de intervenção ${ }^{3}$. Isso nos leva a crer que ainda existem profissionais que realizam o trabalho em grupo sem o devido conhecimento e respaldo teórico, atuando em grupo apenas para vencer a demanda, por meio de uma terapia individualizada, mas com vários indivíduos ao mesmo tempo. Nesses casos, as potencialidades do grupo não são exploradas, e é possível que os resultados não sejam favoráveis.

Alguns pesquisadores vêm fazendo opções teóricas que os respaldam no trabalho fonoaudiológico em grupo. Tais trabalhos se filiam a perspectivas teóricas de campos como sócio-interacionista e interacionista ${ }^{5-8}$, psicologia positiva ${ }^{9}$ e histórico-cultural ${ }^{1,2,10-17}$. Por outro lado há autores que se utilizam de técnicas, mas não assumem teorias de respaldo ${ }^{15}$.

Considerando o exposto acima, o objetivo do presente artigo foi revisar, de maneira sistemática, pesquisas advindas de todas as áreas da Fonoaudiologia que envolveram abordagens grupais, na realidade brasileira.

\section{MÉTODO}

Foi realizado um estudo de revisão sistemática da literatura fonoaudiológica sobre grupo terapêutico. Foi realizada a exploração de artigos, em língua portuguesa, publicados nos bancos de dados das bases SciELO e LILACS no período de 2005 a 2010. Os descritores (DeCS) utilizados para a localização dos artigos foram: audição, voz, saúde pública, linguagem, transtorno de deglutição, fonoterapia, terapêutica, fonoaudiologia, técnicas terapêuticas, reabilitação, promoção da saúde, processos grupais, comunicação, educação em saúde e prática de grupo. A busca foi realizada por descritores isolados e posteriormente pela associação entre eles.

Os critérios de exclusão adotados foram: artigos publicados em idiomas diferentes do português; artigos não publicados em periódicos indexados nas bases de dados LILACS ou SciELO; artigos publicados fora do período de 2005 a 2010; artigos que tratavam de outros tipos de terapia que não a terapêutica grupal. Foram incluídos no estudo artigos originais de pesquisa e de revisão bibliográfica.

Foram selecionados os estudos cujos conteúdos dos resumos relacionavam-se com o objetivo da presente pesquisa. Durante a busca inicial foi encontrado um total de 45 artigos sobre o tema. Considerando-se os critérios de exclusão, foram retirados 17 artigos, restando 28 trabalhos para análise.

Como forma de categorização dos dados, optouse pela análise dos seguintes aspectos: públicoalvo, ano de publicação e área da Fonoaudiologia envolvida. Depois de realizadas as buscas e selecionados os artigos, os dados foram apresentados, sistematicamente, seguindo a ordem cronológica crescente e destacando o posicionamento dos autores com relação à técnica grupal.

\section{REVISÃO DE LITERATURA}

Para apresentação dos dados referentes à revisão, optou-se por fazê-la seguindo a ordem cronológica crescente de publicação dos estudos, já 
que esta é uma das principais categorias de análise. Considerou-se que esta distribuição permite que o leitor acompanhe o histórico das publicações referentes ao grupo terapêutico fonoaudiológico ao longo do tempo. Em seguida, será apresenta a discussão de cada uma das categorias de análise.

Em 2005 foi realizado um estudo com um grupo de pais de crianças deficientes auditivas ${ }^{18}$ buscando investigar as reações e as expectativas deles em relação à patologia apresentada pelo filho e a evolução do caso. Os autores concluíram que o grupo terapêutico propicia o contato com outros pais na mesma condição, o que foi compreendido como necessidade para os pais. Além disso forneceu aos participantes apoio, orientação fonoaudiológica e possibilidade de compartilhar sentimentos, dúvidas e anseios. Ainda em relação à terapêutica grupal familiar, outros estudos permitiram observar que várias das necessidades apresentadas pelas famílias passaram a ser (re)significadas e traduzidas, no contexto terapêutico. Tais resultados indicam que o envolvimento da família pode repercutir positivamente sobre a constituição do sujeito no âmbito social, pois é possível que sejam revistas e modificadas concepções e sentimentos até então vivenciados por ele ${ }^{10,11}$.

Já no âmbito do atendimento grupal junto aos próprios sujeitos com dificuldades fonoaudiológicas, foi desenvolvido um estudo com pacientes disártricos ${ }^{19}$ buscando caracterizar e verificar a eficácia do processo terapêutico fonoaudiológico nessa população. Foi realizada avaliação antes e após processo terapêutico, observando-se que o grupo obteve melhora na avaliação objetiva quanto à prosódia, respiração, fonação e articulação. Além disso, durante avaliação subjetiva, os sujeitos referiram melhora em todas as bases motoras trabaIhadas, concluindo assim que há eficácia do processo terapêutico fonoaudiológico grupal em pacientes disártricos. Outro artigo relata a realização de uma pesquisa com crianças e adolescentes de espectro autístico, buscando identificar mudanças após um período de seis meses de terapia fonoaudiológica, comparando os resultados obtidos nos dois grupos. Concluíram que as crianças apresentaram maiores progressos e ressaltaram a importância da inclusão desses pacientes em processos grupais de terapia de linguagem ${ }^{20}$. Outras pesquisas realizadas com crianças de espectro autístico, também ressaltaram a importância da terapia fonoaudiológica em grupo, e, como esperado, concluíram que o fato de o gesto ser acolhido/significado naquele determinado contexto linguístico-interativo, favoreceu a (re)construção da linguagem, da identidade e da subjetividade dessas crianças $^{12,13}$.
No ano de 2006 foi feito um estudo sobre as possibilidades e aplicações dos grupos focais ${ }^{21}$ ressaltando suas contribuições e aplicações na Fonoaudiologia. Os autores concluíram que o grupo se apresenta como importante estratégia fonoaudiológica, podendo ser aplicado nas práticas que envolvem processos diagnósticos, educativos, terapêuticos e avaliativos das intervenções, projetos e programas na área clínico-terapêutica e/ou preventivo-comunitária da Fonoaudiologia.

Outra importante contribuição sobre a pratica grupal foi trazida ao se estudar o desenvolvimento da narrativa ${ }^{22}$ de crianças surdas usuárias de Língua Brasileira de Sinais (LIBRAS). Os resultados da pesquisa revelaram que atividades em grupo são importantes espaços para o desenvolvimento da LIBRAS, e ampliação do uso da linguagem dessas crianças, sendo que nesse estudo, a LIBRAS foi considerada língua privilegiada na construção dos processos de significação das atividades e situações vivenciadas.

Outra pesquisa realizada com crianças de espectro autístico tomou para análise questões do desenvolvimento da linguagem e sua relação com os aspectos sociocognitivos ${ }^{23}$. Os autores estudaram três situações distintas de comunicação, dentre elas a criança em grupo com o coordenador, e puderam observar que existe relação entre a evolução do desempenho sóciocognitivo e o perfil funcional da comunicação na terapia em grupo.

Foram desenvolvidos também estudos acerca da terapia grupal na área de Voz. Um deles envolveu entrevistas realizadas com fonoaudiólogas que atuavam junto a pacientes disfonicos, observando que a própria dinâmica grupal é um aspecto positivo advindo dessa prática ${ }^{4}$. Ainda na área da voz, houve a publicação de uma experiência com grupo de sujeitos laringectomizados totais ${ }^{24}$, reforçando que o trabalho em grupo mostra-se uma alternativa muito interessante para qualquer sujeito com alterações da comunicação, de qualquer origem. Concluíram que a pratica grupal se agiganta quando se trata de sujeitos laringectomizados totais, que se deparam com o desafio de assumir novamente a direção de suas vidas e retomar suas relações interpessoais e profissionais.

Quanto à prática com pacientes deficientes mentais, estudos analisaram as interações dialógicas em um grupo terapêutico fonoaudiológico formado por jovens com deficiência mental ${ }^{25}$. Os resultados indicaram que o grupo configurou-se como um espaço propício para que processos dialógicos aconteçam, destacando o papel fundamental do fonoaudiólogo no direcionamento do diálogo dos jovens, para que estes se apropriem das mais diversificadas estratégias linguístico-cognitivas. 
Ainda no mesmo ano, outro estudo com deficientes mentais buscou verificar a eficácia do treinamento auditivo em grupo ${ }^{26}$, por meio da comparação dos resultados da avaliação de processamento auditivo, antes e após terapia. Houve melhora nas habilidades auditivas dos indivíduos do grupo no momento da avaliação final, principalmente nas habilidades de localização sonora, sequencia e discriminação auditiva.

Em 2007, alguns trabalhos inovaram, mostrando que a efetividade do grupo pode ocorrer não só na reabilitação, mas também na atuação preventiva ${ }^{27}$. O estudo realizado buscou analisar a importância dos grupos para o processo educativo de prevenção e promoção da saúde vocal com crianças e concluiu que grupos de vivência de voz podem se configurar como um espaço social importante para a promoção da saúde. Nesse mesmo contexto, foram feitas análises sobre a importância do grupo fonoaudiológico no apoio e na reabilitação de trabalhadores portadores de PAIR ${ }^{28}$. O estudo destaca que os principais resultados alcançados pelos trabalhadores foram melhora na comunicação, mudança no comportamento ante as dificuldades comunicativas e diminuição na percepção do handicap.

Foi realizado estudo contendo análise dos processos interativos de afásicos no grupo terapêutico fonoaudiológico ${ }^{29}$ e observou-se que há fatores comuns nos grupos, tais como heterogeneidade, subjetividade, imagens que os sujeitos do grupo (afásicos e não afásicos) fazem de si mesmo e do outro. Esse trabalho ainda ressalta que a análise desses fatores é importante para que se entenda a dinâmica do grupo terapêutico fonoaudiológico, porém, sem deixar de lado as singularidades dos sujeitos pertencentes ao grupo.

Ainda em 2007, foi realizada análise para estabelecer o aproveitamento de um grupo de participantes de um curso para pais de crianças deficientes auditivas ${ }^{30}$. Observou-se que houve diferença nas porcentagens médias de acerto no questionário final, aplicado após a intervenção fonoaudiológica grupal, concluindo que os pais podem se beneficiar de intervenções fonoaudiológicas grupais de promoção de saúde. Além disso, os autores sugerem que os grupos sejam organizados considerando-se o grau de escolaridade dos pais, para que se obtenham maiores índices de aproveitamento.

Em 2008, uma pesquisa analisou a eficácia do grupo fonoaudiológico em um processo de formação sobre a prevenção e promoção da saúde desenvolvido com Agentes Comunitários de Saúde ${ }^{8}$. De acordo com os achados, o processo educativo apresentou-se eficiente para tratar os temas propostos pelo grupo e permitiu o empoderamento no nível individual. No mesmo ano, outro estudo buscou analisar o grupo como modalidade de intervenção fonoaudiológica junto a crianças com distúrbios vocais ${ }^{2}$, bem como as possibilidades de atuação que este espaço propicia. Os resultados mostraram construções conjuntas de conhecimentos, repercussão dos conceitos trabalhados em grupo na vida das crianças, o importante papel do terapeuta, as trocas de experiências, modificações na qualidade vocal de crianças com o decorrer do processo, dentre outros. Os autores concluíram que o grupo é um espaço rico e promissor, importante para o desenvolvimento de noções que as crianças têm da voz e de conhecimentos sobre hábitos saudáveis, além de proporcionar modificações na qualidade vocal das crianças.

Ainda na área de voz, houve um trabalho que analisou a efetividade do grupo de vivencia em $v_{0 z}{ }^{31}$. O método utilizado foi a avaliação vocal de professores de uma escola da rede pública de ensino antes e após a participação nos grupos. Pôde-se concluir que após a vivência de voz houve diferença significante no grau de tensão, melhora dos cuidados com a voz e a compreensão dos fatores intervenientes e determinantes das alterações vocais, presentes nas condições e organização do trabalho docente. Tais achados reforçam a afirmação de que ações educativas processuais se caracterizam como importantes espaços de reflexão e de mudança das relações entre o trabalho e a saúde do professor.

$\mathrm{Na}$ área da linguagem, no ano de 2008, foi efetuado um estudo com o objetivo de refletir sobre a formação/construção da identidade de jovens deficientes mentais que participavam de grupo terapêutico fonoaudiológico ${ }^{14}$, analisando o papel da linguagem no processo de construção da imagem que o sujeito faz de si mesmo. Os achados levantaram a hipótese de que para transformar a imagem que os sujeitos deficientes mentais têm de si mesmos são necessárias ações que incidam sobre aqueles que os rodeiam, ou seja, a família, os amigos, os colegas e o grupo social em geral.

No ano de 2009 , foi realizado um estudo sobre a linguagem escrita e a subjetividade no grupo fonoaudiológico ${ }^{6}$, mostrando que o grupo fonoaudiológico constitui-se como um espaço de troca para que os sujeitos estabeleçam uma relação significante com a leitura e a escrita, propiciando condições fundamentais para a (re)significação dos sintomas e para a interação com diversos textos escritos, promovendo mudanças na relação do sujeito com sua linguagem. Novamente no campo da prevenção e promoção da saúde, um estudo sobre grupo de vivência de voz executado com profissionais de um hospital paulista ${ }^{32}$ ressaltou a importância do 
grupo terapêutico fonoaudiológico na promoção de ambientes saudáveis e de processos comunicativos favoráveis à humanização das relações e melhoria do acesso e qualidade no acolhimento e atendimento em saúde.

Em 2010 foi realizada uma análise dos resultados terapêuticos alcançados a partir da introdução de gêneros textuais como funcionamento terapêutico em um grupo de escolares com queixa de distúrbios de aquisição da linguagem escrita ${ }^{7}$. Concluiuse que o trabalho grupal com gêneros textuais facilitou a motivação para ler e escrever e teve como consequência, a melhoria da progressão e coesão textuais, além da adequação dos aspectos formais da escrita como ortografia e pontuação. Segundo os autores, o trabalho em grupo foi essencial para que os integrantes pudessem (re)significar os sentidos atribuídos à queixa a partir de constante diálogo com troca de experiências de situações escolares e familiares.

Ainda no mesmo ano, foi realizado um estudo buscando analisar os sentidos discursivos observados na fala de afásicos participantes de um grupo terapêutico fonoaudiológico ${ }^{5}$, verificando que o discurso desses sujeitos, independentemente das alterações que apresentaram, está impregnado de sentidos verdadeiros e se produzem em decorrência da interação social entre os interlocutores. Concluíram que mesmo com discurso reduzido, os afásicos apresentaram comportamentos e tentativas de interação com o outro no grupo, estabelecendo processos de significação.

O último artigo sobre grupo publicado em 2010 foi um estudo de revisão bibliográfica sobre a efetividade de abordagens grupais na área da Saúde Coletiva no Brasil, mais especificamente na Fonoaudiologia e na Psicologia ${ }^{9}$. O artigo concluiu que em todos os relatos de caso o grupo foi a forma mais eficaz e eficiente de intervenção, em função de seus pressupostos teóricos consistentes.

É recente a descrição do grupo terapêutico fonoaudiológico na literatura, observando-se assim um número restrito de publicações relacionadas ao tema (28 artigos). A Tabela 1 descreve a quantidade de artigos publicados sobre o tema em cada uma das áreas da Fonoaudiologia, para cada um dos anos estudados e, o total de artigos publicados em cada ano.

Tabela 1 - Quantidade de artigos publicados por ano em cada área da Fonoaudiologia

\begin{tabular}{lccccccc}
\hline Área & $\mathbf{2 0 0 5}$ & $\mathbf{2 0 0 6}$ & $\mathbf{2 0 0 7}$ & $\mathbf{2 0 0 8}$ & $\mathbf{2 0 0 9}$ & $\mathbf{2 0 1 0}$ & Total \\
\hline Saúde Coletiva & 0 & 0 & 0 & 1 & 0 & 1 & 2 \\
Audiologia & 1 & 1 & 2 & 0 & 0 & 0 & 4 \\
Linguagem & 6 & 4 & 1 & 1 & 1 & 1 & 14 \\
Voz & 0 & 2 & 2 & 2 & 1 & 0 & 7 \\
Motricidade Orofacial & 0 & 0 & 0 & 0 & 0 & 0 & 0 \\
Disfagia & 0 & 0 & 0 & 0 & 0 & 0 & 0 \\
Fonoaudiologia Escolar & 0 & 0 & 0 & 0 & 0 & 1 & 1 \\
Total/ano & 7 & 7 & 5 & 4 & 2 & 3 & 28 \\
\hline
\end{tabular}

Com base na análise da tabela, observou-se que existe um número muito reduzido de artigos publicados em periódicos sobre o assunto, visto que o mesmo é um método recente na fonoaudiologia.

Verificou-se que a área de Linguagem tem o maior número de publicações envolvendo grupos, seguida pelas áreas de Voz e Audiologia. Por outro lado, as áreas de Disfagia e da Motricidade Orofacial não foram contempladas em nenhum dos artigos publicados nesse período.

Já em relação ao ano de publicação, observouse que, de maneira geral, tem havido decréscimo do número de publicações sobre o assunto desde o ano de 2007, principalmente na área de Linguagem. Isso pode ter ocorrido pelo fato de muitos profissionais terem uma concepção equivocada sobre o grupo terapêutico, realizando na verdade um agrupamento de indivíduos com alguma característica comum $^{9}$ deixando de lado o acolhimento e o processo de significação das atividades e das situações vivenciadas ${ }^{12,13,22}$.

Embora a área de linguagem tenha maior número de publicações, estes números foram decrescendo com o passar dos anos. Este fato nos chama atenção visto que tem ficado cada vez mais evidente que o grupo terapêutico é um ambiente 
muito rico, de troca de informações entre os pacientes e que permite que as dificuldades sejam (re)significadas e superadas ${ }^{12,13}$.

Pensando na linguagem como o mediador das interações sociais ${ }^{(37)}$, e visto que ela está diretamente relacionada ao pensamento, aos processos cognitivos, ao modo de organizar e categorizar o mundo, desempenhando um papel imprescindível na construção da subjetividade, ela se torna o elo entre os participantes do grupo, e as mudanças possíveis. É por meio da linguagem que os participantes do grupo reconstroem o próprio 'eu', e é nela que os grupos terapêuticos se apoiam para ensinar a própria definição do sujeito. Sendo assim, deve-se levar em conta o papel crucial que a linguagem desempenha em nossas vidas ${ }^{26}$. Ela está direta ou indiretamente envolvida nos grupos terapêuticos fonoaudiológicos e, por isso, deveria ser mais valorizada nas publicações sobre o tema, visto que ela está ligada às significações verbais e não verbais.

$\mathrm{Na}$ Tabela 2 pode-se observar uma análise sobre o público alvo do grupo terapêutico para cada um dos anos estudados.

Tabela 2 - Relação entre público alvo e quantidade de publicações por ano

\begin{tabular}{lcccccccc}
\hline \multirow{2}{*}{ Público Alvo } & $\mathbf{2 0 0 5}$ & $\mathbf{2 0 0 6}$ & $\mathbf{2 0 0 7}$ & $\mathbf{2 0 0 8}$ & $\mathbf{2 0 0 9}$ & $\mathbf{2 0 1 0}$ & \multicolumn{2}{c}{ Total } \\
\cline { 2 - 9 } & $\mathbf{N}$ & $\mathbf{N}$ & $\mathbf{N}$ & $\mathbf{N}$ & $\mathbf{N}$ & $\mathbf{N}$ & $\mathbf{N}$ & $\%$ \\
\hline Pais /Familiares & 3 & 0 & 1 & 0 & 0 & 0 & 4 & 14,28 \\
Pacientes adultos & 2 & 3 & 1 & 2 & 1 & 0 & 9 & 32,15 \\
Pacientes crianças & 1 & 2 & 1 & 2 & 0 & 1 & 7 & 25 \\
Pacientes adolescentes & 1 & 2 & 1 & 0 & 1 & 0 & 5 & 17,85 \\
Pacientes idosos & 0 & 0 & 1 & 0 & 0 & 1 & 2 & 7,14 \\
Nenhum dos grupos & 0 & 0 & 0 & 0 & 0 & 1 & 1 & 3,58 \\
\hline
\end{tabular}

Legenda: $\mathrm{N}=$ número de artigos; \%= porcentagem de artigos

Observa-se que a maior parte dos trabalhos foi realizada com público adulto, seguido de grupos de crianças, adolescentes e idosos, respectivamente. $\mathrm{Na}$ maioria dos artigos, a terapêutica fonoaudiológica grupal vem sendo voltado para o sujeito, intercalando sessões de aconselhamento familiar no decorrer do processo terapêutico.

Pode-se observar que no ano de 2005 houve mais publicações sobre o trabalho terapêutico grupal envolvendo a família. O enfoque com tal população diminuiu nos anos seguinte, e nos três últimos pode-se observar que não houve nenhum trabalho cientifico voltado para esse grupo específico.

Em relação aos trabalhos voltados para o próprio sujeito com dificuldades fonoaudiológicas, pode-se observar que houve mais trabalho nos anos de 2005 e 2006 com pacientes adultos. Nos anos seguintes não houve diferença entre o número de trabalhos voltados para esse público e para os demais e no último ano pesquisado não foi publicado nenhum trabalho com pacientes adultos.

O fato da população de adultos ser mais a mais contemplada nos artigos de grupo é relevante e contraditório, visto que as crianças, por estarem em fase de desenvolvimento, formam grande parte da demanda da clínica fonoaudiológica, e é justamente nessa faixa etária em que as (re)significações delas enquanto sujeitos e reestruturação de sua fala, são mais importantes ${ }^{18}$. E nesse processo o grupo é fundamental.

Outro dado importante que a tabela 2 nos fornece é com relação ao publico alvo da terapia grupal, ou seja, familiares, pais e pacientes. Em 2005 foram publicados 3 estudos com familiares ou pais; já no ano de 2007 houve apenas 1 estudo. Nos demais anos nenhum trabalho com grupo de familiares foi divulgado nas bases pesquisadas, o que nos leva a concluir que no decorrer do tempo o grupo terapêutico ficou mais direcionado para o próprio sujeito.

A preocupação com o papel da família nos grupos terapêuticos é antiga ${ }^{1,2,30,33}$, e mesmo estudos mais recentes incluem o aconselhamento familiar, como um dos pontos principais de evolução terapêutica, visto que muitos deles podem apresentar dificuldades em cuidar de uma criança com algum tipo de patologia fonoaudiológica. Nesse sentido, o grupo fornece à família um espaço onde eles compartiIham sentimentos e histórias de vida ${ }^{10,11,33}$. A família é importante também, porque, muitas vezes, é o único referencial para o paciente, antes de ele passar a frequentar a clínica fonoaudiológica fornecendo dados a respeito das vivências do paciente $e$ 
passando a ser um facilitador no processo de recuperação do sujeito ${ }^{33}$.

Apenas um estudo não se enquadrou nos critérios de classificação, sendo ele uma revisão bibliográfica $^{9}$ de grupo terapêutico fonoaudiológico e psicológico na área da saúde coletiva.

Apesar de longe de ser considerado um processo terapêutico tradicional e intensamente descrito cientificamente, a terapia grupal no âmbito da fonoaudiologia vem evidenciando seu benefício no processo de (re)constituição do sujeito que passa a ter uma nova visão sobre si, sobre o outro, sobre as relações sociais, sobre as dificuldades e, especialmente, sobre as próprias condições de linguagem e comunicação.

\section{CONCLUSÃO}

Conclui-se que existe um número reduzido de artigos publicados em periódicos sobre o assunto.

Quanto aos objetivos do trabalho grupal, verificou-se que a fonoaudiologia vem inovando, propondo também grupos fonoaudiológicos que visam a prevenção e promoção da saúde.

Conclui-se ainda que a maior parte dos trabaInos foi realizada com público adulto, seguido de grupos de crianças, adolescentes e idosos, respectivamente. Na maioria dos artigos, a terapêutica fonoaudiológica grupal vem sendo voltada para o sujeito, intercalando sessões de aconselhamento familiar no decorrer do processo terapêutico. O índice de artigos envolvendo apenas grupos de familiares foi baixo.

Dentre as áreas da Fonoaudiologia, verificouse que a de Linguagem tem o maior número de publicações em terapêutico grupal, seguida pelas áreas de Voz e Audiologia. Já em relação ao ano de publicação, observa-se que, de maneira geral, tem havido decréscimo do número de publicações sobre o assunto desde o ano de 2007, principalmente na área de Linguagem.

Considera-se que além de novos estudos sobre a prática grupal, outros trabalhos de revisão devam assumir a análise de categorias como aspectos metodológicos, estratégias de atuação e resultados obtidos nos processos terapêuticos grupais.

O grupo possibilita uma nova visão sobre o próprio sujeito e sobre o outro, devido à diversidade de experiência e conhecimentos compartilhados nas sessões, diminuindo o isolamento, o peso e ansiedade criada pela patologia em torno do sujeito e de sua família.

\begin{abstract}
Background: therapeutic Group in Speech Language and Hearing Sciences. Purpose: to review, systematically, researches stemming from all areas of speech therapy that involved group approaches, in Brazil. We conducted a search in LILACS and SciELO databases in the period from 2005 to 2010. We selected studies where the contents of the abstracts were related to the objective of this research. As a way of categorizing such data, we chose to analyze the following aspects: target audience, year of publication and area of Speech Language and Hearing Sciences. Conclusion: there are a limited number of publications concerning the topic (28 articles). Most studies were conducted with adult audiences, followed by groups of children, adolescents and elderly, respectively. The index of articles involving family groups was low. Among the areas of Speech, Language has the largest number of publications involving groups, followed by the areas of Speech and Audiology. As for the year of publication, we noted that, in general, there has been a decline in the number of publications on the subject since the year 2007. We may conclude then that the number of publications on the therapeutic group, is restricted, in the area of Speech Language and Hearing Sciences. We believe that in addition to new studies on the group practice, other revision works should assume the analysis of the categories, such as methodological issues, strategies and performance results obtained in the group-related therapeutic processes.
\end{abstract}

KEYWORDS: Group Processes; Speech, Language and Hearing Sciences; Speech Therapy; Group Practice; Therapeutics 


\section{REFERÊNCIAS}

1. Leite APD, Panhoca I. A constituição de sujeitos no grupo terapêutico fonoaudiológico: identidade e subjetividade no universo da clínica fonoaudiológica. Rev Dist Comun. 2003; 15(2):289-308.

2. Leite APD, Panhoca I, Zanolli ML. Distúrbios de voz em crianças: o grupo como possibilidade de intervenção. Rev Dist Comun. 2008; 20(3):339-47.

3. Lopes JC. O vínculo e sua relevância no trabalho terapêutico fonoaudiológico com grupos [dissertação]. São Paulo (SP): Pontifícia Universidade Católica de São Paulo; 2008.

4. Vilela FCA, Ferreira LP. Voz na clínica fonoaudiológica: grupo terapêutico como possibilidade. Dist Comun. 2006; 18(2):235-43.

5. Albuquerque AG, Costa MLG, Sena EFC, Luz LMS. Análise da produção de sentidos em narrativas de afásicos participantes de grupo de convivência. Rev. CEFAC [online]. 2010 Nov; 12(1): 51-6.

6. Machado MLCA, Berberian AP, Santana AP. Linguagem escrita e subjetividade: implicações do trabalho grupal. Rev. CEFAC [online]. 2009: 11(4):713-9.

7. Schneider ACB, Souza APR, Deuschle VP. Intervenção fonoaudiológica com gêneros textuais em um grupo de escolares. Rev. CEFAC [online]. 2010; 12(2):337-45.

8. Brites LS, Souza APR, Lessa AH. Fonoaudiólogo e agente comunitário de saúde: uma experiência educativa. Rev. Soc. Bras. Fonoaudiol. [online]. 2008; 13(3):258-66.

9. Souza APR, Crestani AH, Vieira CR, Machado FCM, Pereira LL. O grupo na fonoaudiologia: origens clínicas e na saúde coletiva. Rev. CEFAC [online]. 2010; 12(3):200-9.

10. Penteado RZ, Panhoca I, Siqueira D, Romano FF, Lopes P. Grupalidade e família na clínica fonoaudiológica: deixando emergir a subjetividade. Rev Dist Comun. 2005; 17(2):161-71.

11. Monteiro MIB, Camargo EAA, Freitas AP, Bagarollo MF. Interações dialógicas de familiares de sujeitos com deficiência mental: algumas reflexões. Temas Desenvolv. 2005-6; 14(83-84):32-9.

12. Zia J, Panhoca I, Zanolli ML. O acolhimento da gestualidade na terapia de linguagem: reflexões no âmbito da clínica fonoaudiológica. Rev Dist Comun. 2005; 17(3):365-72.

13. Panhoca I, Camargo EAA, Freitas AP, Monteiro MIB. O grupo terapêutico-fonoaudiológico e o processo de construção da identidade e da subjetividade. Fono Atual. 2005; 8(31):53-5.

14. Leite GA, Monteiro MIB. A construção da identidade de sujeitos deficientes no grupo terapêutico-fonoaudiológico. Rev. bras. educ. espec. [online]. 2008; 14(2):189-200.
15. Bagarollo MF; Panhoca, I. A constituição da subjetividade de adolescentes autistas: um olhar para as histórias de vida. Revista Brasileira de Educação Especial. 2010; 16: 231-50.

16. Guimaraes MF, Behlau MS, Panhoca I. Análise perceptivo-auditiva da estabilidade vocal de adolescentes em diferentes tarefas fonatórias. Pró-Fono. 2010; 22: 455-68.

17. Panhoca I. Grupo terapêutico-fonoaudiológico: aprofundando um pouco mais as reflexões. Distúrbios da Comunicação. 2007; 19: 257-62.

18. Boscolo CC, Santos TMM. A deficiência auditiva e a família: sentimentos e expectativas de um grupo de pais de crianças com deficiência da audição. Rev Dist Comun. 2005; 17(1):69-75.

19. Miranda CS, Soares ECS, Ortiz KZ. Eficácia do processo terapêutico fonoaudiológico em grupo para disartria. Fono Atual. 2005; 8(32):32-9.

20. Fernandes FD. Resultados de terapia fonoaudiológica com adolescentes com diagnóstico inserido no espectro autístico. Pró-Fono. 2005;17(1):67-76.

21. Penteado RZ, Gonçalves CGO, Silvério KCA, Rossi D, Libardi A, Vieira TPG. Grupos Focais: possibilidades e aplicações para as pesquisas e práticas fonoaudiológicas. Rev Soc Bras Fonoaudiol. 2006;11(2):124-8.

22. Lacerda CBF, Lodi ACB. O desenvolvimento do narrar em crianças surdas: o contexto de grupo e a importância da língua de sinais. Temas Desenvolv. 2006; 15(85-56):45-53.

23. Cardoso C, Fernandes FDM. Relação entre os aspectos sócio cognitivos e perfil funcional da comunicação em um grupo de adolescentes do espectro autístico. Pró-Fono R. Atual. Cient. [online]. 2006; 18(1):89-98.

24. Mourão LF, Servilha EAM, Mercuri AAS, Beilke $\mathrm{HMB}$, Xavier PE. Grupo terapêutico fonoaudiológico desenvolvido junto a laringectomizados totais: uma experiência em situação de clínica-escola. Rev Dist Comun. 2006; 18(1):51-61.

25. Freitas AP, Castro GS. A constituição de processos dialógicos em um grupo de jovens com deficiência mental. Rev. bras. educ. espec. [online]. 2006; 12(1):49-64.

26. Santos JN, Couto ICP, Amorim RMC. Treinamento auditivo em oficinas: opção terapêutica grupal. Pró-Fono. 2006; 18(3):293-302.

27. Penteado RZ, Camargo AMD, Rodrigues CF, Silva CR, Rossi D, Silva JTC, et al. Vivência de voz com crianças: análise do processo educativo em saúde vocal. Rev Dist Comun. 2007; 19(2):237-46.

28. Gonçalves CGO. Análise do programa de apoio e reabilitação para trabalhadores portadores de PAIR em uma metalúrgica. Rev Dist Comun. 2007; 19(1):103-16. 
29. Fernandes DF. Processos Interativos em Grupo: sujeitos afásicos no grupo. Rev Dist Comum. 2007; 19(2):276-7.

30. Moret ALM, Freitas VS, Ferreira MCF, Alvarenga $\mathrm{KF}$, Bevilacqua MC. Curso de pais de crianças deficientes auditivas: estudo do conhecimento dos pais em um módulo intermediário. Rev Dist Comun. 2007; 19(1):25-37.

31. Silverio KCA, Gonçalves CGO, Penteado RZ, Vieira TPG, Libardi A, Rossi D. Actions in vocal health: a proposal for improving the vocal profile of teachers. Pró-Fono Revista de Atualização Científica. 2008 jul-set;20(3):177-82.
32. Penteado RZ, Stenico E, Ferrador FA, Anselmo NC, Silva PC, Peirera PFA et al. Vivência de voz com profissionais de um hospital: relato de experiência. Rev. CEFAC [online]. 2009; 11(3):449-56.

33. Santana AP, Dias F, Serrato MRF. O afásico e seu cuidador: discussões sobre um grupo de familiares. In: Santana AP, Berberian AP, Guarinello AP, Massi G (org.) Abordagens Grupais em Fonoaudiologia: contextos e aplicações. São Paulo: Plexus, 2007, p. 11-38.

http://dx.doi.org/10.1590/S1516-18462011005000131

RECEBIDO EM: 29/03/2011

ACEITO EM: 07/06/2011

Endereço para correspondência:

Vanessa Veis Ribeiro

Rua Souza Naves, 227, Jardim Califórnia

Irati - PR

CEP: 84500-000

E-mail: vanessaribeirooo@hotmail.com 